Revista de

Contabilidade e

Organizações
DOI: http://dx.doi.org/10.11606/rco.v10i27.111708
Journal of

Accounting and

Organizations

\title{
Nova metodologia do Ibovespa, betas e poder explicativo dos retornos das ações
}

\author{
Ricardo Goulart Serra ${ }^{\mathrm{a}, \mathrm{c}}$; André Taue Saito ${ }^{\mathrm{b}}$; Luiz Paulo Lopes Fávero ${ }^{\mathrm{c}}$ \\ ${ }^{a}$ Fundação Escola de Comércio Álvares Penteado \\ ${ }^{b}$ Universidade Federal de São Paulo \\ ${ }^{c}$ Universidade de São Paulo
}

\begin{tabular}{l} 
Informações do Artigo \\
\hline Histórico do Artigo \\
Recebido: 02 de março de 2016 \\
Aceito: 17 de agosto de 2016 \\
\hline Palavras chave: \\
Ibovespa. \\
Retorno. \\
Beta. \\
\hline
\end{tabular}

\section{INTRODUÇÃO}

\begin{abstract}
Resumo
Em 2013, houve mudança na metodologia do Ibovespa, implementada a partir de 2014. A BM\&FBOVESPA divulgou uma série histórica retroativa de 34 trimestres do Ibovespa calculado com a metodologia nova (Ibovespa Novo) em paralelo ao obtido com a metodologia anterior (Ibovespa Antigo). O objetivo do artigo é verificar se o beta calculado regredindo o retorno das ações contra o Ibovespa Novo (BetaNovo) teria sido capaz de explicar melhor do que o beta calculado regredindo o retorno das ações contra o Ibovespa Antigo (BetaAntigo). O modelo em painel com efeitos fixos é, segundo os testes apropriados, preferível ao modelo POLS e ao de efeitos aleatórios, e indica que o BetaAntigo teria sido capaz de explicar o retorno melhor do que o BetaNovo. O modelo explica melhor a variação entre observações e explica pouco a variação de uma mesma ação ao longo do tempo. O fato de, no período analisado, ter existido apenas o Ibovespa Antigo pode ter influenciado os resultados.
\end{abstract}

Copyright (C) 2016 FEA-RP/USP. Todos os direitos reservados

Um dos temas, que é muito estudado em finanças, é a relação entre o beta e o retorno das ações. Pelo CAPM - capital asset pricing model (SHARPE, 1964; LINTNER, 1965 e BLACK, 1972), o beta mede todo o risco não diversificável e, portanto, seria o único parâmetro necessário para explicar o retorno. Os testes empíricos, no entanto, verificam se o beta é capaz de, sozinho, explicar o retorno das ações. As evidências empíricas, neste sentido, são fracas (MALKIEL, 2014). A alternativa é acrescentar outras variáveis explicativas que meçam ou tenham hipotética relação com o risco, por meio de modelos multifatoriais, visando melhorar a explicação do retorno das ações com estas variáveis adicionais, na presença ou não do beta.

Diversos estudos têm documentado melhora na explicação do retorno das ações por meio de modelos multifatoriais. Pode-se citar, como exemplo, o modelo de três fatores de Fama e French (1993 e 1996) que considera, além do beta, o tamanho (representado pelo valor de mercado do patrimônio líquido) e o índice Book to Market (valor contábil dividido pelo valor de mercado) como fatores explicativos do retorno das ações. O modelo de quatro fatores de Carhart (1997), por sua vez, adiciona momento, além das variáveis do modelo de três fatores, na explicação do retorno. A liquidez também é lembrada nos testes, tendo Acharya e Pedersen (2005) incluído-a no seu modelo, em conjunto com tamanho e Book to Market, e Liu (2006) utilizado-a acompanhada da variável momento. Mais recentemente, Fama e French (2015) especificaram um modelo de cinco fatores acrescentando, ao modelo de três fatores, a rentabilidade e o investimento.

Um dos motivos para o beta eventualmente não explicar o retorno de maneira isolada é que ele não mede todo o risco não diversificável, ou seja, ele é uma medida incompleta do risco. No entanto, outro motivo é a inadequação de sua estimação. Tal inadequação pode ser gerada, dentre outros motivos expostos na fundamentação teórica, pelo índice de mercado utilizado na estimação do beta. O índice de mercado usado na estimação do beta deveria refletir todos os ativos existentes. Como não existe índice que meça todos os ativos existentes, é comum utilizar um índice de bolsa como proxy para o índice de mercado. No entanto, nada garante que índices de bolsa sejam carteiras eficientes (THOMÉ et al., 2011), sendo senso comum que estejam longe da fronteira eficiente (LEVY; ROLL, 2010).

Autor Correspondente: Tel (11) 3203-0133

E-mail: ricardo.serra@usp.br (R. G. Serra); andretauesaito@gmail.com (A. T. Saito); 1pfavero@usp.br (L. P. L. Fávero)

Fundação Escola de Comércio Álvares Penteado - Av. da Liberdade, 532, Liberdade, São Paulo - SP, 01502-001, Brasil. 
No Brasil, é comum utilizar o índice Bovespa como proxy do índice de mercado, embora hajam críticas à utilização de um índice local para tal finalidade (HAGLER; BRITO, 2007 e PADMANABAN et al., 2013). Alguns autores questionam a capacidade do Ibovespa refletir a economia brasileira, visto que apresenta problemas metodológicos (SHENG; SAITO, 2002; TAKAMATSU; LAMOUNIER, 2006 e RABELO, 2007). Outros estudaram se o Ibovespa pode ser considerado uma carteira eficiente, tendo alguns deles concluído negativamente (THOMÉ et al., 2011 e SERRA; NAKAMURA, 2016) e outros de forma inconclusiva ou positivamente (NAKAMURA, 2000 e NODA et al., 2014). Portanto, existem argumentos que indicam que o fato de o beta não explicar de maneira isolada o retorno das ações brasileiras pode ser, pelo menos parcialmente, devido a utilização do Ibovespa como proxy do índice de mercado na estimação do beta.

A recente mudança na metodologia para inclusão de ações na carteira do Ibovespa (anunciada no final de 2013 e implantada em etapas a partir de 2014) levanta obrigatoriamente a pergunta: o beta estimado a partir do Ibovespa formado conforme a nova metodologia (BetaNovo) seria capaz de explicar melhor o retorno das ações do que o beta estimado a partir do Ibovespa formado conforme a metodologia antiga (BetaAntigo)? Dessa forma, a hipótese de pesquisa consiste em verificar se o BetaNovo teria sido capaz de explicar melhor o retorno das ações brasileiras comparativamente ao BetaAntigo.

A amostra é composta pelas ações integrantes do Ibovespa Antigo (Ibovespa formado conforme a metodologia antiga) e do Ibovespa Novo (Ibovespa formado conforme a metodologia nova). O período considerado na análise é de 01 de janeiro de 2003 a 30 de abril de 2014, período para o qual se tem a série histórica do Ibovespa Novo, divulgado retroativamente pela BM\&FBOVESPA (2016b), além da série histórica do Ibovespa Antigo. No total, estudou-se 118 ações ao longo de 34 trimestres. O poder de explicação foi medido pelo coeficiente de ajuste da regressão, conhecido por $\mathrm{R}^{2}$.

O presente artigo contribui com o estudo do Ibovespa Novo, pouco investigado até o presente momento. Mais especificamente, documenta indícios de que, no período analisado, o beta produzido pelo Ibovespa Novo não foi capaz de explicar melhor o retorno das ações brasileiras do que o beta produzido pelo Ibovespa Antigo.

Além desta introdução, o artigo contém as seguintes seções: fundamentação teórica, metodologia, análise de dados e considerações finais.

\section{FUNDAMENTAÇÃO TEÓRICA}

\subsection{Beta como medida de risco}

Desde que Markowitz (1952) colocou o risco em igualdade com o retorno na tomada de decisão, o mesmo tem sido bastante investigado. A evolução do seu trabalho por Sharpe (1964), Lintner (1965) e Black (1972) culminou na medida de risco conhecida por beta, que, diferente do desvio padrão (medida de risco total), propõe-se a medir o risco não diversificável. Pela formulação do CAPM - capital asset pricing model, que é um modelo de um único fator, o beta seria a única variável necessária na explicação do retorno.

Na sequência, os estudos buscaram identificar, por meio de modelos multifatoriais, se o beta mede todo o risco não diversificável. Os testes incluíam, além do beta, outras variáveis explicativas para o retorno. É sabido que tais testes não são capazes de distinguir se (a) a medida de risco é inadequada ou incompleta - no caso, o beta ou (b) se o mercado é ineficiente (MALKIEL, 2014), trata-se de um teste conjunto de ambas as hipóteses.

Os primeiros testes somavam ao beta outra variável, tais como: (a) tamanho (BANZ, 1981), (b) índice Book to Market (STATMAN, 1980), (c) alavancagem (BHANDARI, 1988) e (d) liquidez (AMIHUD; MENDELSON, 1986). Chan et al. (1991) e Fama e French (1992) testaram-nas em conjunto para verificar potencial redundância, tendo Fama e French (1992 e 1993) formulado o modelo de três fatores: beta, tamanho e Book to Market (ou prêmios ligados a estes fatores), para explicar o retorno.

As discussões que se seguiram buscavam entender se tais variáveis eram (i) medidas de risco, (ii) proxies de medidas de risco não observáveis ou (iii) representavam alguma anomalia do mercado. Caso trate-se de uma anomalia, o mercado aprenderá com ela e a mesma não persistirá. Portanto, seus resultados devem ser interpretados com cuidado (BANZ, 1981).

Seria tamanho uma medida, direta ou indireta, de risco (empresas pequenas tendo maior risco)? Existem 
outras hipóteses para justificar sua significância na explicação do retorno, tais como: (i) os índices de bolsa, utilizados para calcular o beta, são majoritariamente compostos por empresas grandes e, portanto, capazes de explicar o retorno das empresas grandes, sendo necessário outro fator para explicar o retorno das empresas pequenas (BANZ, 1981), (ii) o beta das empresas pequenas, por serem ilíquidas, podem ter sido calculados enviesados para baixo (ROLL, 1981) ou (iii) fundos de pensão e seguradoras têm limitação (econômica e operacional) para investir em empresas pequenas, fazendo com que estas estejam subavaliadas por serem negligenciadas (BLACK, 1993).

Book to Market é uma medida de risco (direta ou indireta)? Outra explicação para sua significância está ligada a hipóteses comportamentais (LAKONISHOK et al., 1994): os investidores sobrevalorizam os resultados históricos (positivos ou negativos) embutindo no preço atual das ações suas expectativas sobrevalorizadas que, em não acontecendo, acabam impactando os preços no futuro. Por exemplo, empresas com bons crescimentos históricos são precificadas com estes bons crescimentos extrapolados para o futuro, portanto, sendo precificadas com preços 'altos e 'baixos índices' Book to Market. No decorrer do tempo, ao não repetirem suas boas atuações do passado, têm suas expectativas recalibradas para baixo, o mesmo acontecendo com seus preços. Segundo este raciocínio, empresas com baixos índices Book to Market tenderiam a render menos do que o esperado.

O mesmo pode-se dizer de alavancagem financeira ou liquidez - seriam realmente medidas de risco (direta ou indireta)? O risco pela alavancagem financeira já deveria estar presente no beta, porém, sendo o último calculado por meio de uma série histórica, pode não refletir a alavancagem atual, tornando-a importante na explicação do retorno (BHANDARI, 1988). Igualmente, o maior retorno encontrado para ações com baixa liquidez pode não ser devido ao maior risco destas ações, mas pelo maior spread bid-ask dos preços das mesmas, o que exige delas um retorno bruto maior como compensação deste custo de transação, resultando em mesmo retorno líquido que aquele observado para ações líquidas (AMIHUD; MENDELSON, 1986).

Modelos posteriores, como o de Carhart (1997), incluíram novas variáveis a serem investigadas, como o momento. Normalmente são dois os efeitos encontrados ligados a momento: (i) um efeito de curto prazo, no qual as ações com bons(maus) retornos passados continuam tendo bons(maus) retornos por um curto prazo e (ii) um efeito de longo prazo, no qual as ações com bons(maus) retornos sofrem uma reversão de retornos. Para o curto prazo, as hipóteses que embasam tais comportamentos são: (a) o entusiasmo dos investidores e (b) a lenta resposta do mercado a nova informação, por exemplo, bons resultados não são de imediato incorporados ao preço, mas lentamente, implicando em um período de retornos anormais. Já o efeito de longo prazo pode ter explicação comportamental: sobre reação dos investidores (MALKIEL, 2014).

Acadêmicos preocupados em analisar se o mercado lê e aprende com tais estudos passaram a medir o retorno anormal após a publicação dos mesmos. Os resultados são conflitantes: (a) Jegadeesh e Titman (2001) documentaram evidências de que os efeitos ligados ao momento aumentaram (maiores retornos anormais) e (b) Schwert (2003) evidenciaram o contrário para variáveis como tamanho e book to market. A utilização de outras variáveis explicativas do retorno, que não o beta, pela indústria de fundos, parece indicar não haver dúvida de que os investidores aprendem com a academia. Segundo Malkiel (2014), tais variáveis são usadas para escolher ativos que deem sabor ("flavour") à carteira, favorecendo o seu retorno comparativamente ao benchmark. No entanto, estas carteiras "nem sempre rendem mais do que o mercado e quando o fazem, não passam no teste de risco" (MALKIEL, 2014, p. 127). Ao se concentrarem em ativos com as características desejadas agregam, pelo menos, mais risco diversificável (JACOBS; LEVY, 2014).

Recentemente, McLean e Pontiff (2016) investigaram 97 características tidas como explicativas do retorno, encontradas em 79 diferentes artigos acadêmicos. Investigaram o retorno anormal (1) na amostra (mesmo período de tempo relatado nos artigos originais), (2) posterior à amostra (entre o período após o término do período da amostra e antes da publicação do artigo) e (3) posterior à publicação do artigo. Encontraram queda no retorno anormal do período 1 para o período 2, o que indica (a) potencial data mining ou outro tipo de falha metodológica ou (b) que o investidor aprendeu sobre a estratégia mesmo antes da publicação do artigo. Documentaram queda no retorno anormal do período 1 para o período 3, superior à queda do período 1 para o período 2 , sendo a queda residual estatisticamente diferente de zero, mas não total a ponto de eliminar o retorno anormal produzido pela estratégia. Esta queda residual pode ser interpretada como um indício de que o efeito produzido pela estratégia é derivado de má precificação ou anomalia, e não por risco maior. Caso o efeito derivasse de risco, não deveriam encontrar queda residual do retorno entre os períodos 1 e 3, pois a publicação do artigo não revelaria "informação que induziria agentes racionais a terem comportamento diferente” (MCLEAN; PONTIFF, 2016, p. 7).

Portanto, parece não haver consenso sobre as demais variáveis explicativas do retorno serem medidas de risco (direta ou indireta). O beta, diferentemente das demais variáveis, foi deduzido teoricamente (as demais são testadas empiricamente). Sendo assim, sobrevive mesmo que como medida de risco potencialmente incompleta, uma vez que, como já mencionado, os testes empíricos envolvendo a explicação do retorno pelo beta não testa 
exclusivamente a validade do beta como medida de risco, mas também (i) a capacidade de estimá-lo corretamente (decorrente de decisões importantes no seu cálculo, conforme seção 2.2) e (ii) a eficiência do mercado.

Sendo assim, entende-se que é crucial o entendimento sobre quais parâmetros são utilizados na construção do beta, incluindo a escolha do índice de mercado, aproximado por um índice de bolsa. Neste sentido, o presente artigo compara o poder de explicação do beta produzido a partir do Ibovespa Antigo com o poder de explicação do beta produzido a partir do Ibovespa Novo.

\subsection{Decisões metodológicas no cálculo do Beta}

O beta de uma ação pode ser calculado regredindo o retorno da ação contra o retorno do mercado, como na Equação (1) ou na sua variante conhecida como market model exposto na Equação (2), adotada no presente artigo:

$$
\begin{gathered}
R_{i, t}=R_{f, t}+\beta_{i}\left(R_{m, t}-R_{f, t}\right)+\varepsilon_{i} \\
R_{i, t}=\alpha_{i}+\beta_{i} R_{m, t}+\varepsilon_{i}
\end{gathered}
$$

Em que:

$\mathrm{R}_{\mathrm{i}, \mathrm{t}}$ é o retorno da ação i no período $t, \mathrm{R}_{\mathrm{f}, \mathrm{t}}$ é o retorno do ativo livre de risco no período $\mathrm{t}, \mathrm{R}_{\mathrm{m}, \mathrm{t}}$ é o retorno do mercado no período t, $\alpha_{i}$ é o intercepto da ação i, $\beta_{\mathrm{i}}$ é o beta da ação e $\boldsymbol{\varepsilon}_{\mathrm{i}}$ é o termo de erro com média zero e desvio padrão constante.

Além da decisão do modelo a ser adotado, existem três decisões, de natureza metodológica, para a estimação do beta de uma ação: qual (i) o índice de bolsa adequado, se global ou local; (ii) o histórico de retornos apropriado, em anos; e (iii) a periodicidade de retorno condizente, se diária, semanal ou mensal.

Quanto ao índice de bolsa, o investidor globalizado é mais bem servido com um índice global. Damodaran (1994) e Koller et al. (2005) sugerem o MSCI World Index, em consonância com Copeland et al. (2002) que, no entanto, por considerarem que os índices globais são relativamente recentes, recorrem ao índice da bolsa de Nova Iorque (Estados Unidos) - S\&P 500. No entanto, é comum o emprego de um índice de bolsa local, ou seja, do país onde a sede da empresa é localizada - no caso do Brasil, o Ibovespa. Para atender o objetivo do artigo, a opção foi utilizar o Ibovespa.

No que se refere ao histórico de retornos, (a) por um lado o maior número de observações resulta em melhor precisão e, consequentemente, menor erro de estimativa do beta, (b) por outro lado o maior número de observações (no caso, um histórico com mais anos) aumenta a probabilidade de ocorrência de mudanças estruturais na empresa com impacto no seu risco, o que compromete a representatividade do beta. Daves et al. (2000) indicam a utilização de um histórico de até 3 anos após constatarem que mais de 50\% das firmas sofrem mudanças significativas de risco em 3 anos. Desta forma, considerando-se que a janela de tempo escolhida deva possibilitar o maior número de observações sem comprometer a representatividade do beta, optou-se pelo emprego, no presente estudo, de um período de três anos de histórico de retornos (ou o maior existente).

Por fim, no que diz respeito à periodicidade dos retornos, Daves et al. (2000) seguem a linha de o maior número de observações ser melhor e indicam a periodicidade de retorno diária, por resultar menor erro padrão na estimativa de betas. No entanto, a utilização de periodicidade diária enviesa o beta de ações com baixa liquidez (DIMSON,1979, SCHOLES; WILLIAMS, 1977 e SERRA; MARTELANC, 2013). Uma alternativa para correção do viés é aumentar a periodicidade (HANDA et al., 1989; DAMODARAN, 1997 e KOLLER et al., 2005).

Além do aumento da periodicidade, existem alternativas para minimizar o viés de betas de ações de baixa liquidez, ajustando-se as estimativas por dois métodos alternativos ao método base de Repetição da Última Cotação - RUC: (i) Trade-to-Trade - TT; e (ii) ajuste de Scholes e Williams (1977) - SW (SERRA; MARTELANC, 2013). No RUC, atribui-se para uma data em que a ação não foi negociada a cotação da última data em que a mesma foi negociada. Tal procedimento reduz a covariância do retorno da ação com o retorno do mercado com o consequente viés para baixo dos betas estimados de ações com baixa liquidez. No TT, são utilizadas apenas datas em que a ação negociou - supondo que o índice de mercado tem cotação diária (MARSH, 1979, DIMSON; MARSH, 1983 e SERRA; MARTELANC, 2013). No procedimento SW, o beta é calculado considerando betas estimados com os retornos defasados e adiantados, como variáveis independentes adicionais (SCHOLES; WILLIAMS, 1977), 
conforme Equação (3):

$$
\begin{gathered}
\beta=\frac{\operatorname{cov}\left(r_{i, t}, r_{m, t}\right)}{\operatorname{var}\left(r_{m, t}\right)} \\
\beta_{\text {adiantado }}=\frac{\operatorname{cov}\left(r_{i, t}, r_{m, t+1}\right)}{\operatorname{var}\left(r_{m, t+1}\right)} \\
\beta_{S W}=\frac{\beta_{\text {defasado }}+\beta+\beta_{\text {adiantado }}}{1+2 \cdot \operatorname{corr}\left(r_{m, t}, r_{m, t+1}\right)}
\end{gathered}
$$

Em que:

$\beta_{\mathrm{SW}}$ é o beta calculado conforme o ajuste proposto por Scholes e Williams (1977), $\beta_{\text {defasado }}$ o beta calculado com a série de retorno do mercado defasada em relação à série de retorno da ação, $\beta$ é o beta calculado com as duas séries de retornos considerando o mesmo período de tempo, $\beta_{\text {adiantado }}$ é o beta calculado com a série de retorno do mercado adiantada em relação à série de retorno da ação, $r_{i}$ é o retorno da ação e $r_{m}$ é o retorno do mercado. $\mathrm{O}$ período atual é representado por $\mathrm{t}$, o período defasado por $\mathrm{t}-1$ e o período adiantado por $\mathrm{t}+1$.

No conjunto das alternativas, este artigo trabalhou com o market model e o cenário base composto de: (a) índice local (objeto do artigo) - Ibovespa, (b) histórico de retorno de 3 anos (histórico máximo sem comprometer a representatividade do beta), (c) periodicidade diária e (d) ajuste por SW.

\subsection{Ibovespa, sua importância e suas limitações}

O Ibovespa é o principal índice da Bolsa de Valores de São Paulo (BM\&FBovespa), configurando-se em sua definição como "o indicador de desempenho médio das cotações dos ativos de maior negociabilidade e representatividade do mercado de ações brasileiro" (BM\&FBOVESPA, 2016a).

Não obstante sua importância, o Ibovespa sempre foi bastante criticado. A alta concentração de certas ações no índice, o critério de composição ser fortemente baseado na liquidez e os diferentes critérios para manutenção e inclusão de ações costumam ser fonte de parte das críticas (SHENG; SAITO, 2002; TAKAMATSU; LAMOUNIER, 2006 e RABELO, 2007). Outra crítica, que independe dos critérios estabelecidos pela BM\&FBOVESPA, é que algumas empresas grandes não são negociadas na bolsa, o que acarreta na subrepresentação de setores importantes da economia na composição do Ibovespa (SERRA; WICKERT, 2014). Um exemplo é o setor automotivo. Portanto, parece haver indícios de que o Ibovespa peca como representante da economia brasileira.

Outra linha de pesquisa visa identificar se o Ibovespa é uma carteira eficiente. NAKAMURA (2000) e NODA et al. (2014) indicam não haver motivos para rejeitar tal hipótese. Outros autores documentam carteiras que teriam sido superiores ao Ibovespa (BRUNI; FAMÁ, 1998; HAGLER; BRITO, 2007; THOMÉ et al., 2011; SANTOS; TESSARI, 2012 e SERRA; NAKAMURA, 2016). Os índices de outros países também são testados quanto a sua eficiência, com resultados favoráveis (LEVY; ROLL, 2010) e desfavoráveis (DEMIGUEL et al., 2009 e PADMANABAN et al., 2013).

Aparentemente motivada a corrigir algumas distorções do Ibovespa, a BM\&FBovespa divulgou, em setembro de 2013, nova metodologia de cálculo do índice a ser implementada integralmente até maio de 2014. Tais modificações impactam as regras (i) para a inclusão no índice (aumento do índice de negociabilidade, alteração no critério de participação nos pregões e não ser "penny stock" ou estar listada como "situação especial") e (ii) de definição do peso de cada ação no índice, para implicar maior importância ao valor total negociado, e de estabelecimento de um limite de participação.

Tais mudanças justificam revisitar determinados testes dada a importância do Ibovespa e seus diversos desdobramentos, inclusive no proposto pelo presente artigo: o cálculo do beta, essencial em análises financeiras e estudos acadêmicos. 


\section{METODOLOGIA}

\subsection{Amostra}

A composição das carteiras do Ibovespa (Antigo ou Novo) entre janeiro de 2003 e janeiro de 2014 foi obtida em BM\&FBOVESPA (2016b). No total, a amostra considera 118 ações que compuseram pelo menos uma das carteiras (Ibovespa Antigo ou Ibovespa Novo) nos 34 quadrimestres analisados. Os retornos do Ibovespa Antigo e do Ibovespa Novo também foram obtidos em BM\&FBOVESPA (2016b).

Os preços (ajustados pelos proventos) das 118 ações foram obtidos no sistema de informação Economática ${ }^{\circledR}$ (pelo período de análise). Algumas ações mudaram de nome durante o período de análise, e neste trabalho foram consideradas as mudanças indicadas na Tabela 1.

Tabela 1. Mudança de nome de determinadas ações ao longo do período analisado

\begin{tabular}{cccc}
\hline Nome Original & Nome Atual & Nome Original & Nome Atual \\
\hline BMEF3 & BVMF3 & LLXL3 & PRML3 \\
BRTO4 & OIBR4 & PCAR5 & PCAR4 \\
CLSC6 & CLSC4 & PRGA3 & BRFS3 \\
CESP4 & CESP5 & VCPA3 & FIBR3 \\
ECOD3 & VAGR3 & TLPP4 & VIVT4 \\
ELPL6 & ELPL4 & TSPP4 & VIVO4 \\
ITAU4 & ITUB4 & TCSL3 & TIMP3 \\
\hline
\end{tabular}

Fonte: Elaborada pelos autores.

\subsection{Cálculo do Beta}

O beta foi calculado regredindo o retorno de cada uma das ações contra o retorno de cada um dos índices (Ibovespa Novo e Ibovespa Antigo), conforme o market model. Como Cenário Base, utilizou-se o histórico de retornos de 3 anos e a periodicidade diária conforme indicado por Daves et al. (2000). Para evitar problemas de falta de liquidez, realizou-se o ajuste por Scholes e Williams (1977). Como Cenário Alternativo foram calculados betas para histórico de retornos 2 anos e periodicidade semanal. Calculou-se também, como cenário alternativo, os betas sem os ajustes de Scholes e Williams (1977).

Desta forma, calcularam-se, para cada um dos índices, os seguintes 'cenários' de betas: (1) histórico de 3 anos e periodicidade diária, com ajuste de Scholes e Williams (SW), (2) histórico de 3 anos e periodicidade diária, sem ajuste de SW, (3) histórico de 3 anos e periodicidade semanal, com ajuste de SW, (4) histórico de 3 anos e periodicidade semanal, sem ajuste de SW, (5) histórico de 2 anos e periodicidade diária, com ajuste de SW, (6) histórico de 2 anos e periodicidade diária, sem ajuste de SW, (7) histórico de 2 anos e periodicidade semanal, com ajuste de SW e (8) histórico de 2 anos e periodicidade semanal, sem ajuste de SW.

\subsection{Procedimento}

Para atingir o objetivo de verificar se o beta calculado regredindo o retorno das ações contra o Ibovespa Novo (BetaNovo) teria sido capaz de explicar melhor o retorno das ações comparativamente ao beta calculado regredindo o retorno das ações contra o Ibovespa Antigo (BetaAntigo), é necessário um banco de dados composto de período (meses), observações (ações), betas (para os diversos cenários) e retornos mensais.

Tome-se como exemplo o Cenário Base de beta calculado com 3 anos de histórico de retornos e periodicidade diária. Para se ter um histórico de 3 anos dentro do período de análise de 01 de janeiro de 2003 a 30 de abril de 2014, tomou-se como primeira observação mensal o período iniciado em 01 de janeiro de 2006. A estimação do beta deu-se no período de 01 de janeiro de 2003 a 31 de dezembro de 2005 (retornos diários) para 
explicar o retorno do mês subsequente, de 01 de janeiro de 2006 a 31 de janeiro de 2006. A última observação mensal, neste cenário, iniciou-se em 01 de abril de 2014, tendo como período de estimação 31 de março de 2011 a 31 de março de 2014 para explicar o retorno do mês subsequente, de 01 de abril de 2014 a 30 de abril de 2014. No total, neste exemplo, tem-se 100 meses de observação no banco de dados (mesmo número para a periodicidade semanal). Para o histórico de retornos de 2 anos, foram 112 meses de observação no banco de dados. Em cada um dos meses de observação foram analisadas as ações que compunham o Ibovespa Antigo ou o Ibovespa Novo. Por exemplo, para o mês de observação de junho de 2010, foram analisadas as ações que compunham o Ibovespa Antigo ou o Ibovespa Novo no quadrimestre de 01 de maio de 2010 a 31 de agosto de 2010. A Tabela 2 apresenta a quantidade de ações analisadas por quadrimestre.

Tabela 2. Quantidade de ações analisadas por quadrimestre

\begin{tabular}{lllclc}
\hline Quadrimestre & Qtde de Ações & Quadrimestre & Qtde de Ações & Quadrimestre & Qtde de Ações \\
\hline jan-03 & 41 & set-06 & 51 & mai-10 & 57 \\
mai-03 & 39 & jan-07 & 54 & set-10 & 58 \\
set-03 & 37 & mai-07 & 57 & jan-11 & 58 \\
jan-04 & 39 & set-07 & 61 & mai-11 & 60 \\
mai-04 & 40 & jan-08 & 66 & set-11 & 63 \\
set-04 & 42 & mai-08 & 66 & jan-12 & 62 \\
jan-05 & 44 & set-08 & 59 & mai-12 & 65 \\
mai-05 & 47 & jan-09 & 55 & set-12 & 64 \\
set-05 & 48 & mai-09 & 52 & jan-13 & 66 \\
jan-06 & 49 & set-09 & 51 & mai-13 & 69 \\
mai-06 & 51 & jan-10 & 54 & set-13 & 73 \\
& & & & jan-14 & 72 \\
\hline
\end{tabular}

Fonte: Elaborada pelos autores.

Para cada mês, tomou-se (a) o retorno da ação quando o retorno do mercado foi positivo (medido pelo índice, o Ibovespa Antigo ou o Ibovespa Novo, conforme o caso) e (b) o retorno da ação multiplicado por -1 quando o retorno do mercado foi negativo. Tal procedimento foi realizado para que os coeficiente angulares tenham o mesmo sinal em ambas as situações (meses com prêmio de mercado positivo e meses com prêmio de mercado negativo). Conforme pode ser visto na Figura 1, (1) em meses que o mercado teve retorno positivo (valores esperados sob o contexto de prêmio de mercado positivo), seria esperado que quanto maior(menor) o beta de uma dada ação, maior(menor) o retorno desta. Assim, o coeficiente angular da regressão do retorno da ação contra o beta deveria ser positivo (linha sólida da Figura 1). (2) Em meses que o mercado teve retorno negativo (valores esperados sob o contexto de prêmio de mercado negativo), seria esperado que quanto maior(menor) o beta de uma dada ação, menor(maior) o retorno desta mesma ação, assim, o coeficiente angular da regressão beta x retorno deveria ser negativo (linha pontilhada da Figura 1). A primeira vista pode parecer estranho, mas a interpretação é de maior(menor) beta implicar retornos mais(menos) negativos, no sentido literal de perda.

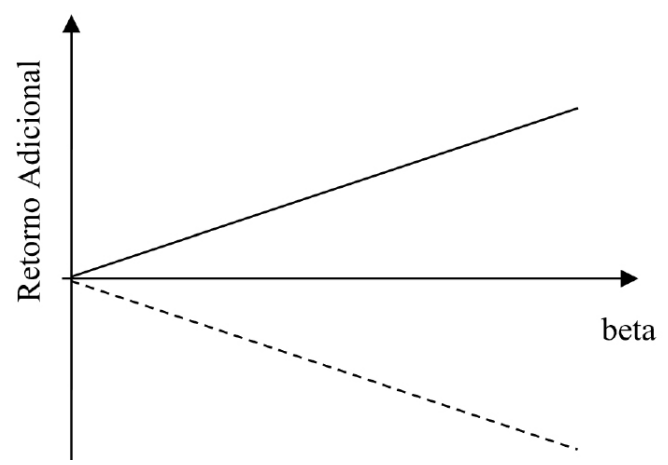

Figura 1. Beta $x$ Retorno Adicional (acima do retorno livre de risco)

Nota: Em teoria, o retorno adicional deve (i) aumentar conforme o beta aumenta em períodos de alta do mercado (linha sólida) e (ii) cair conforme o beta aumenta em períodos de baixa do mercado (linha pontilhada).

Fonte: Elaborada pelos autores. 


\subsection{Dados em Painel}

Como a amostra é composta de até 118 observações (ações) por até 112 períodos (períodos mensais), utilizou-se (i) a regressão POLS (pooled ordinary least squares) e (ii) dados em painel com efeitos fixos (fixed effects) e efeitos aleatórios (random effects). Trata-se de um painel desbalanceado (nem todas as observações estão presentes em todos os períodos) e curto (mais observações do que períodos). Foram utilizados modelos robustos para atenuar problemas decorrentes de heterocedasticidade. O teste de Breusch-Pagan e o teste de Hausman foram aplicados para verificação do melhor modelo. Segundo Fávero (2015), é fundamental a aplicação do teste robusto de Hausman, também descrito por Hoechle (2007) e Cameron e Trivedi (2009). O teste de Schaffer e Stillman (2010), equivalente ao teste robusto de Hausman (FÁVERO, 2015), também foi aplicado. O teste de BreuschPagan testa a hipótese nula de que não há efeito em painel, o teste de Hausman testa a hipótese nula de que o efeito é aleatório (sendo a hipótese alternativa de que o efeito é fixo) e o teste de Schaffer e Stillman também testa a hipótese nula de que o efeito é aleatório (sendo a hipótese alternativa de que o efeito é fixo).

\section{ANÁLISE DE DADOS}

\subsection{Análise Descritiva}

A Tabela 3 apresenta as estatísticas descritivas dos retornos para os cenários de estimação de beta (diferentes históricos e periodicidades). Foram eliminados da amostra os valores superiores a 2,58 desvios-padrões e inferiores a 2,58 desvios-padrões de todas as variáveis.

Tabela 3. Estatísticas descritivas

\begin{tabular}{|c|c|c|c|c|c|c|}
\hline Cenário & Variável & Mínimo & Média & Máximo & Desv Pad & Qtde Obser \\
\hline \multirow[t]{5}{*}{2 anos / diária } & Retorno & $-45,2 \%$ & $0,9 \%$ & $32,0 \%$ & $9,6 \%$ & 6.339 \\
\hline & BetaAntigo & 0,1536 & 0,8947 & 2,0364 & 0,2885 & \\
\hline & BetaAntigo SW & 0,1233 & 0,9011 & 1,9028 & 0,3204 & \\
\hline & BetaNovo & 0,1823 & 0,8998 & 1,9294 & 0,2754 & \\
\hline & BetaNovo SW & 0,1255 & 0,8853 & 1,7424 & 0,3044 & \\
\hline \multirow[t]{5}{*}{2 anos / semanal } & Retorno & $-45,2 \%$ & $0,9 \%$ & $32,0 \%$ & $9,6 \%$ & 6.257 \\
\hline & BetaAntigo & $-0,0767$ & 0,8830 & 2,4224 & 0,3351 & \\
\hline & BetaAntigo SW & $-0,3276$ & 0,8846 & 3,4350 & 0,4198 & \\
\hline & BetaNovo & $-0,0729$ & 0,8728 & 2,4844 & 0,3289 & \\
\hline & BetaNovo SW & $-0,5266$ & 0,8684 & 3,7873 & 0,4212 & \\
\hline \multirow[t]{5}{*}{3 anos / diária } & Retorno & $-27,9 \%$ & $0,7 \%$ & $29,2 \%$ & $9,5 \%$ & 5.767 \\
\hline & BetaAntigo & 0,1590 & 0,8875 & 1,6257 & 0,2730 & \\
\hline & BetaAntigo SW & 0,0955 & 0,8963 & 1,7510 & 0,3084 & \\
\hline & BetaNovo & 0,1877 & 0,8849 & 1,5831 & 0,2604 & \\
\hline & BetaNovo SW & 0,0817 & 0,8739 & 1,6407 & 0,2930 & \\
\hline \multirow[t]{5}{*}{3 anos / semanal } & Retorno & $-27,9 \%$ & $0,8 \%$ & $29,2 \%$ & $9,5 \%$ & 5.686 \\
\hline & BetaAntigo & $-0,0146$ & 0,8664 & 1,7714 & 0,3238 & \\
\hline & BetaAntigo SW & $-0,1826$ & 0,8742 & 2,0790 & 0,3984 & \\
\hline & BetaNovo & $-0,0128$ & 0,8492 & 1,7038 & 0,3123 & \\
\hline & BetaNovo SW & $-0,2476$ & 0,8507 & 1,9591 & 0,3873 & \\
\hline
\end{tabular}

Nota: Mínimo, Média, Máximo e Desvio Padrão para cada variável: Retorno, BetaAntigo (beta calculado a partir do Ibovespa Antigo), BetaAntigo SW (idem, mas com ajuste de Scholes e Williams), BetaNovo (beta calculado a partir do Ibovespa Novo) e BetaNovo SW (idem, mas com ajuste de Scholes e Williams), para cada Cenário indicado por histórico de retorno / periodicidade (por exemplo, 2 anos / diária). A coluna observação indica o número de observações para cada cenário.

Fonte: Elaborada pelos autores. 
É interessante analisar o comportamento das variáveis (dependente e explicativa) ao longo do tempo e entre indivíduos. Neste sentido, calculou-se a variação within (variações para cada indivíduo ao longo do tempo) - que indica mudanças da variável de cada indivíduo ao longo do tempo, a variação between (variações entre indivíduos) - que indica diferença de comportamento entre os indivíduos e a variação overall (variação de um indivíduo em determinado período comparativamente aos demais em todos os períodos) - que é decomposta nas duas anteriores (Fávero, 2015). A Tabela 4 apresenta essas variações.

Tabela 4. Decomposição da variação das variáveis

\begin{tabular}{|c|c|c|c|c|}
\hline Cenário & Variável & Overall & Between & Within \\
\hline \multirow[t]{5}{*}{2 anos / diária } & Retorno & 0,09602 & 0,02510 & 0,09502 \\
\hline & BetaAntigo & 0,28853 & 0,28227 & 0,16663 \\
\hline & BetaAntigo SW & 0,32040 & 0,29832 & 0,18324 \\
\hline & BetaNovo & 0,27543 & 0,25774 & 0,15690 \\
\hline & BetaNovo SW & 0,30439 & 0,26995 & 0,17075 \\
\hline \multirow[t]{5}{*}{2 anos / semanal } & Retorno & 0,09588 & 0,02412 & 0,09485 \\
\hline & BetaAntigo & 0,33509 & 0,30749 & 0,20891 \\
\hline & BetaAntigo SW & 0,41978 & 0,37533 & 0,28188 \\
\hline & BetaNovo & 0,32889 & 0,28858 & 0,20576 \\
\hline & BetaNovo SW & 0,42119 & 0,36234 & 0,28329 \\
\hline \multirow[t]{5}{*}{3 anos / diária } & Retorno & 0,09520 & 0,02402 & 0,09417 \\
\hline & BetaAntigo & 0,27304 & 0,26787 & 0,14929 \\
\hline & BetaAntigo SW & 0,30842 & 0,28592 & 0,15920 \\
\hline & BetaNovo & 0,26037 & 0,24681 & 0,13584 \\
\hline & BetaNovo SW & 0,29300 & 0,25972 & 0,14340 \\
\hline \multirow[t]{5}{*}{3 anos / semanal } & Retorno & 0,09482 & 0,02454 & 0,09378 \\
\hline & BetaAntigo & 0,32377 & 0,30162 & 0,18819 \\
\hline & BetaAntigo SW & 0,39843 & 0,36243 & 0,24080 \\
\hline & BetaNovo & 0,31233 & 0,28468 & 0,17607 \\
\hline & BetaNovo SW & 0,38730 & 0,34920 & 0,22972 \\
\hline
\end{tabular}

Nota: Variação Overall (variação de um indivíduo em determinado período comparativamente aos demais períodos), Between (variação entre indivíduos) e Within (variação para cada indivíduo ao longo do tempo) para cada variável: Retorno, BetaAntigo (beta calculado a partir do Ibovespa Antigo), BetaAntigo SW (idem, mas com ajuste de Scholes e Williams), BetaNovo (beta calculado a partir do Ibovespa Novo) e BetaNovo SW (idem, mas com ajuste de Scholes e Williams), para cada Cenário indicado por histórico de retorno / periodicidade (por exemplo, 2 anos / diária).

Fonte: Elaborada pelos autores.

Analisando a Tabela 4, observa-se que a maior variação da variável retorno é within, ou seja, de uma mesma ação ao longo do tempo - sendo aproximadamente 4 vezes maior do que a variação between. No que diz respeito à variável explicativa (diversos betas), a variação between é maior. O fato da variável retorno ter maior variação within é indício de que o modelo de efeitos fixos pode ser o mais indicado. Embora a variação between seja maior para os betas, esperava-se uma variação within ainda menor - a variação within é proporcionalmente alta comparativamente a variação between (ou seja, esperava-se uma maior estabilidade do beta de uma mesma ação ao longo do tempo). Esta magnitude da variação within pode ser decorrente do erro de estimação dos betas e/ou por mudança de classe de risco ao longo do tempo. Nota-se que as variações são maiores para os betas SW.

Estas mesmas conclusões podem ser observadas na Figura 2, em que são apresentados - a título de exemplificação apenas para o cenário de beta calculado com o Ibovespa Novo, histórico de 2 anos, periodicidade diária e sem ajuste de SW - os gráficos para cada observação e ao longo do tempo do retorno (painel (a)) e beta (painel (b), respectivamente). O painel (a) indica que o retorno tem uma variação maior entre observações ao longo do tempo (within) e o painel (b) indica que o beta tem uma variação maior entre uma mesma observação ao longo do tempo (between). 


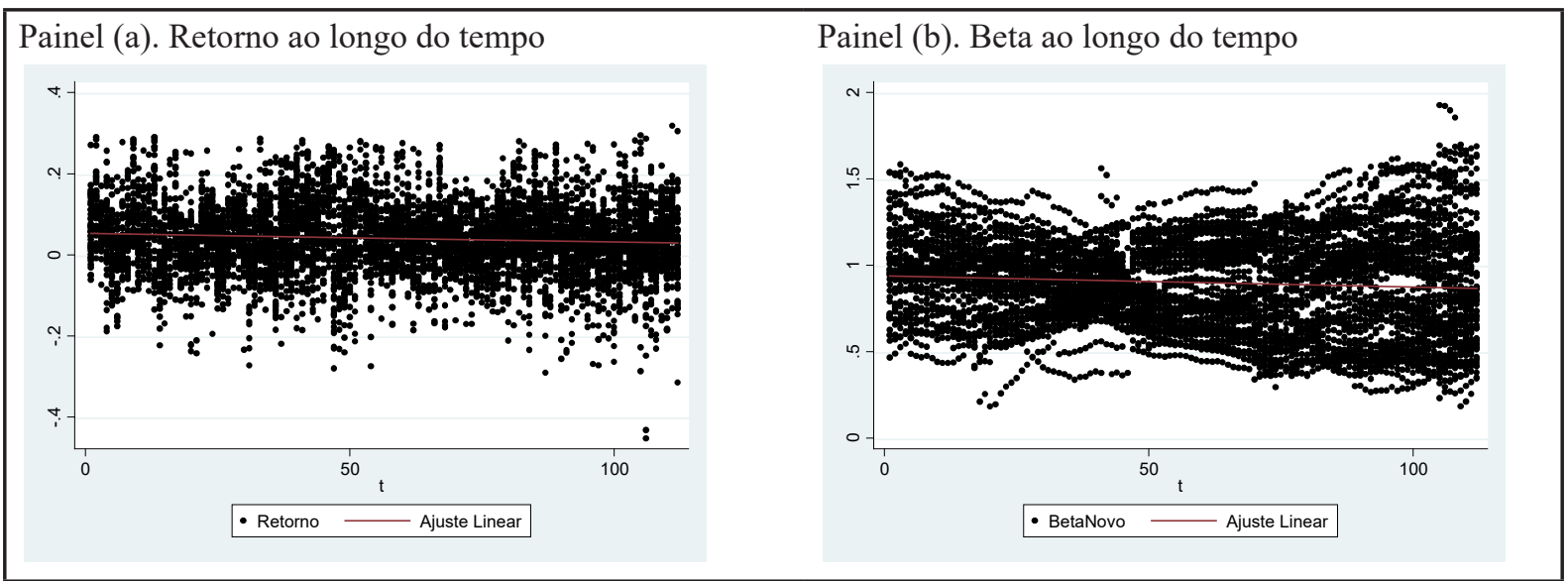

Figura 2. Evolução do retorno (painel (a)) e do beta (painel (b)) ao longo do tempo, por observação

Nota: O cenário de beta foi o com o Ibovespa Novo, histórico de 2 anos, periodicidade diária e sem ajuste de SW, a título de exemplificação dos diversos cenários.

Fonte: Elaborada pelos autores em Stata ${ }^{\circledR}$.

\subsection{Coeficientes de Ajuste $\mathbf{R}^{2}$}

Os testes mencionados na seção 3.4 indicam que o modelo de efeitos fixos é o mais adequado, prevalecendo sobre o modelo POLS e sobre o modelo de efeitos aleatórios. Este resultado corrobora a suspeita de que este seria o melhor modelo ao analisar-se a variação da variável dependente (Tabela 4). Os $\mathrm{R}^{2}$ do modelo POLS são iguais aos $\mathrm{R}^{2}$ overall apresentados e os $\mathrm{R}^{2}$ do modelo de efeitos aleatórios são iguais aos $\mathrm{R}^{2}$ dos modelos de efeitos fixos e não são apresentados. Os $\mathrm{R}^{2}$ dos modelos de efeitos fixos (para cada cenário) estão apresentados na Tabela 5. O painel (a) apresenta os $\mathrm{R}^{2}$ overall, o painel (b) apresenta os $\mathrm{R}^{2}$ between e o painel (c) apresenta os $\mathrm{R}^{2}$ within. Embora não apresentados, todos os coeficientes angulares dos betas (em cada cenário) são significativos a 1\%.

Tabela 5. $\mathrm{R}^{2}$ do modelo de efeitos fixos, para cada cenário de beta

Painel (a): R ${ }^{2}$ overall

\begin{tabular}{lllcc}
\hline Histórico & Periodicidade & SW & Ibovespa Antigo & Ibovespa Novo \\
\hline 2 anos & Diária & Sim & $2,3 \%$ & $1,6 \%$ \\
2 anos & Diária & Não & $1,9 \%$ & $1,2 \%$ \\
2 anos & Semanal & Sim & $1,9 \%$ & $1,4 \%$ \\
2 anos & Semanal & Não & $1,8 \%$ & $1,3 \%$ \\
3 anos & Diária & Sim & $2,5 \%$ & $1,6 \%$ \\
3 anos & Diária & Não & $2,1 \%$ & $1,3 \%$ \\
3 anos & Semanal & Sim & $2,6 \%$ & $1,9 \%$ \\
3 anos & Semanal & Não & $2,1 \%$ & $1,3 \%$ \\
\hline
\end{tabular}

Painel (b): $\mathrm{R}^{2}$ between

\begin{tabular}{lllcc}
\hline Histórico & Periodicidade & SW & Ibovespa Antigo & Ibovespa Novo \\
\hline 2 anos & Diária & Sim & $18,7 \%$ & $16,9 \%$ \\
2 anos & Diária & Não & $19,6 \%$ & $17,7 \%$ \\
2 anos & Semanal & Sim & $22,8 \%$ & $17,8 \%$ \\
2 anos & Semanal & Não & $12,2 \%$ & $8,9 \%$ \\
3 anos & Diária & Sim & $19,2 \%$ & $13,8 \%$ \\
3 anos & Diária & Não & $23,7 \%$ & $17,9 \%$ \\
3 anos & Semanal & Sim & $22,8 \%$ & $17,4 \%$ \\
3 anos & Semanal & Não & $12,4 \%$ & $6,6 \%$ \\
\hline
\end{tabular}


Painel (c): $\mathrm{R}^{2}$ within

\begin{tabular}{lllcc}
\hline Histórico & Periodicidade & SW & Ibovespa Antigo & Ibovespa Novo \\
\hline 2 anos & Diária & Sim & $0,1 \%$ & $0,0 \%$ \\
2 anos & Diária & Não & $0,1 \%$ & $0,0 \%$ \\
2 anos & Semanal & Sim & $0,1 \%$ & $0,0 \%$ \\
2 anos & Semanal & Não & $0,1 \%$ & $0,0 \%$ \\
3 anos & Diária & Sim & $0,1 \%$ & $0,0 \%$ \\
3 anos & Diária & Não & $0,0 \%$ & $0,1 \%$ \\
3 anos & Semanal & Sim & $0,2 \%$ & $0,0 \%$ \\
3 anos & Semanal & Não & $0,1 \%$ & $0,0 \%$ \\
\hline
\end{tabular}

Nota: O painel (a) apresenta os $\mathrm{R}^{2}$ overall, o painel (b) apresenta os $\mathrm{R}^{2}$ between e o painel (c) apresenta os $\mathrm{R}^{2}$ within. A coluna Histórico apresenta o cenário de histórico de retorno do cálculo do beta ( 2 e 3 anos), a coluna Periodicidade apresenta a periodicidade do retorno no cálculo do beta (diária ou semanal), a coluna SW indica se o beta foi (sim) ou não foi (não) calculado com o ajuste de Scholes e Williams (1977), a coluna Ibovespa Antigo apresenta o R ${ }^{2}$ da regressão com a variável explicativa do retorno sendo o beta calculado regredindo o retorno contra o Ibovespa Antigo (BetaAntigo) e a coluna Ibovespa Novo é similar ao Ibovespa Antigo mas para o Ibovespa Novo.

Fonte: Elaborada pelos autores.

Pela Tabela 5, observa-se que o BetaAntigo teria explicado melhor o retorno do que o BetaNovo. Os $\mathrm{R}^{2}$ (overall, between e within) de todos os cenários (exceto 1 cenário do $\mathrm{R}^{2}$ within) são numericamente maiores para os betas calculados a partir do Ibovespa Antigo. Embora a maior variação do retorno seja within, o modelo foi capaz de explicar melhor a variação between, em linha com a maior variação da variável explicativa (beta). Também pode-se observar, na Tabela 5, que o beta calculado com o ajuste de Scholes e Williams (1977) nem sempre explicou melhor o retorno do que o beta sem o ajuste. Serra e Martelanc (2013) indicam que outros ajustes para betas de empresas com baixa liquidez podem, em determinadas situações, ser superiores ao ajuste de Scholes e Williams (1977). Do ponto de vista do sinal do coeficiente angular da variável beta, os betas calculados com os ajustes de Scholes e Williams (1977) foram mais coerentes, tendo em vista que em 3 cenários estes coeficientes foram negativos (sendo esperado que fossem sempre positivos) para betas calculados sem o ajuste: betas calculados com o Ibovespa Novo para histórico de 2 anos e periodicidade diária, betas calculados com o Ibovespa Novo para histórico de 3 anos e periodicidade diária e betas calculados com o Ibovespa Novo para histórico de 3 anos e periodicidade semanal. O coeficiente angular dos betas calculados com o Ibovespa Novo para histórico de 3 anos, periodicidade diária e com o ajuste também foi negativo. Nenhum coeficiente angular dos betas calculados com o Ibovespa Antigo foi negativo, o que também favorece o Ibovespa Antigo comparativamente ao Novo.

\subsection{Robustez}

O painel (b) da Tabela 5 é abaixo reapresentado para os resultados com os dados antes da exclusão dos outliers (renomeada Tabela 6). Portanto, apresenta-se os $\mathrm{R}^{2}$ between do modelo de efeito fixo (modelo mais indicado segundo os testes da seção anterior). Os coeficientes angulares dos betas de todos os modelos são significantes a $1 \%$. Os $\mathrm{R}^{2}$ within são pequenos comparativamente aos $\mathrm{R}^{2}$ between e por isto não são apresentados.

Tabela 6. $\mathrm{R}^{2}$ between do painel fixed effects

\begin{tabular}{lllcc}
\hline Histórico & Periodicidade & SW & Ibovespa Antigo & Ibovespa Novo \\
\hline 2 anos & Diária & Sim & $23,36 \%$ & $16,50 \%$ \\
2 anos & Diária & Não & $26,71 \%$ & $20,84 \%$ \\
2 anos & Semanal & Sim & $37,94 \%$ & $26,81 \%$ \\
2 anos & Semanal & Não & $18,64 \%$ & $10,61 \%$ \\
3 anos & Diária & Sim & $22,72 \%$ & $14,25 \%$ \\
3 anos & Diária & Não & $28,84 \%$ & $20,67 \%$ \\
3 anos & Semanal & Sim & $25,50 \%$ & $18,12 \%$ \\
3 anos & Semanal & Não & $15,02 \%$ & $7,49 \%$
\end{tabular}

Nota: Idem ao que foi apresentado no painel (b) da Tabela 5, porém, com todos os dados antes da exclusão dos outliers. Fonte: Elaborada pelos autores. 
Pode-se observar pela Tabela 6 que os resultados continuam indicando uma preferência pelo BetaAntigo em relação ao BetaNovo.

A Tabela 7 reapresenta os resultados considerando os próprios retornos a cada mês (sem considerar -1 vezes o retorno quando o retorno do mercado foi negativo). Da mesma forma, apresenta-se os $\mathrm{R}^{2}$ between do painel fixed effects.

Tabela 7. $\mathrm{R}^{2}$ between do painel fixed effects

\begin{tabular}{lllcc}
\hline Histórico & Periodicidade & SW & Ibovespa Antigo & Ibovespa Novo \\
\hline 2 anos & Diária & Sim & $4,86 \%$ & $3,65 \%$ \\
2 anos & Diária & Não & $7,35 \%$ & $5,97 \%$ \\
2 anos & Semanal & Sim & $4,04 \%$ & $4,23 \%$ \\
2 anos & Semanal & Não & $1,02 \%$ & $0,71 \%$ \\
3 anos & Diária & Sim & $3,92 \%$ & $2,61 \%$ \\
3 anos & Diária & Não & $6,37 \%$ & $5,26 \%$ \\
3 anos & Semanal & Sim & $2,82 \%$ & $2,54 \%$ \\
3 anos & Semanal & Não & $0,09 \%$ & $0,01 \%$ \\
\hline
\end{tabular}

Nota: Idem ao que foi apresentado no painel (b) da Tabela 5, porém, considerando o próprio retorno das ações mesmo quando o retorno do mercado foi negativo.

Fonte: Elaborada pelos autores.

Observa-se pela Tabela 7 que o BetaAntigo foi superior ao BetaNovo exceto em um cenário, para o qual os $\mathrm{R}^{2}$ foram praticamente iguais.

\section{CONSIDERAÇÕES FINAIS}

No final de 2013 foram anunciadas modificações na metodologia para inclusão de ações na carteira do Ibovespa. Estas modificações foram implantadas em etapas a partir de 2014. A BM\&FBOVESPA (2016b) divulgou o Ibovespa Novo retroativamente de 01 de janeiro de 2003 a 30 de abril de 2014 - período de análise. O objetivo deste artigo é verificar se o beta calculado regredindo o retorno das ações contra o Ibovespa Novo teria sido capaz de explicar melhor o retorno das ações comparativamente ao beta calculado regredindo o retorno das ações contra o Ibovespa Antigo. Foram testadas 118 ações que compuseram o Ibovespa (Antigo ou Novo) ao longo do período de análise. Os betas calculados a partir do Ibovespa Novo são estatisticamente diferentes dos betas calculados a partir do Ibovespa Antigo (valor-p do teste de média emparelhado é 0,000 para todos os cenários).

A análise da variação das variáveis indicou que os retornos têm uma variação de uma mesma ação ao longo do tempo (variação within) aproximadamente quatro vezes maior do que a variação entre ações (variação between). Esta constatação indica que o modelo de efeitos fixos pode ser mais pertinente para sua análise. A variável explicativa (beta), por sua vez, apresentou uma variação entre ações (between) maior do que a variação de uma mesma ação ao longo do tempo (within). No entanto, a variação within ainda é significativa (frente a variação between), o que pode ser devido a erro de estimação do beta e/ou devido a mudança do perfil de risco das empresas ao longo do tempo, em linha com Daves et al. (2000).

Os testes dos modelos estimados indicaram a pertinência dos modelos de efeitos fixos (mais adequados do que o modelo POLS ou o modelo de efeitos aleatórios). Os $\mathrm{R}^{2}$ between mostraram-se significativamente maiores do que os $\mathrm{R}^{2}$ within, indicando que o modelo tem um poder de explicação maior para as variações entre ações. Estes resultados podem corroborar o pensamento de que as variações do retorno das ações ao longo do tempo seriam explicadas pelas variações do retorno do mercado ao longo do tempo (efeito within), enquanto as variações entre ações em cada período de tempo seriam explicadas pelos diferentes betas (ou níveis de risco).

Comparando o coeficiente de ajuste $\mathrm{R}^{2}$ dos modelos em painel, pode-se verificar indícios de que o beta calculado tendo como índice de mercado o Ibovespa Antigo teve um poder de explicação do retorno maior do que aquele do beta calculado tendo como índice de mercado o Ibovespa Novo. É importante destacar que, durante o período analisado, existiu apenas o Ibovespa Antigo. O Ibovespa Novo utilizado nas análises foi calculado 
retroativamente. Pode-se pensar que o retorno das ações seja influenciado pelo retorno do mercado, no caso, o Ibovespa Antigo. Sendo assim, o fato de ter existido apenas o Ibovespa Antigo durante o período de análise pode ter favorecido o poder de explicação dos betas calculados a partir do Ibovespa Antigo. O baixo $\mathrm{R}^{2}$ overall de ambos os betas (Novo e Antigo) está em linha com Malkiel (2014, p. 129) quando afirma que os testes empíricos não dão suporte a teoria de que quanto maior o beta (risco) maior deve ser o retorno da ação.

A restrição do artigo é analisar apenas o período de 01 de janeiro de 2003 a 30 de abril de 2014 (no entanto, é a única janela possível de análise) e o fato de o Ibovespa Novo não ter existido de fato durante o período de análise.

\section{REFERÊNCIAS}

ACHARYA. V. V.; PEDERSEN, L. H. Asset pricing with liquidity risk. Journal of Financial Economics, v. 77, n. 2, p. 375-410, 2005.

AMIHUD, Y.; MENDELSON, H. Liquidity and stock returns. Financial Analysts Journal, v. 42, n. 3, p. 43-48, 1986.

BANZ, R. W. The relationship between return and market value of common stocks. Journal of Financial Economics, v. 9, n. 1, p. 3-18, 1981.

BHANDARI, L. C. Debt/equity ratio and expected common stock returns: empirical evidence. Journal of Finance, v. 43, p. 507-528, 1988

BLACK, F. Capital Market Equilibrium with Restricted Borrowing. Journal of Business, v. 45, n. 3, p. 444-455, 1972.

BLACK, F. Beta and return. Journal of Portfolio Management, v. 20, p. 8-18, 1993.

BM\&FBOVESPA. O que é o Ibovespa?, 2016a. <Disponível em http://www.bmfbovespa.com.br/indices/ ResumoIndice.aspx? Indice=Ibovespa\&idioma=pt-br $>$ Acesso em fevereiro de 2016.

BM\&FBOVESPA. Série retroativa do Ibovespa com base na metodologia adotada em Setembro de 2013. 2016b < Disponível em http://www.bmfbovespa.com.br/Indices/download/SERIE-RETROATIVA-DO-IBOVMETODOLOGIA-VALIDA-A-PARTIR-09-2013.pdf> Acesso em fevereiro de 2016.

BRUNI, A. L.; FAMÁ, R. Moderna teoria de portfólios: É possível captar, na prática, os benefícios decorrentes de sua utilização? Resenha da BM\&F, n. 128, p. 19-34, 1998.

CAMERON, A. C.; TRIVEDI, P. K. Microeconometrixs using Stata. Revised editions. College Station: Stata Press, 2009.

CARHART, M. M. On persistence in mutual fund performance. Journal of Finance, v. 52, n. 1, p. 57-82, 1997.

CHAN, L. K.; HAMAO, Y.; LAKONISHOK, J. Fundamentals and stock returns in Japan. Journal of Finance, v. 46, n. 5, p. 1739-1789, 1991.

COPELAND, T.; KOLLER, T.; MURRIN, J. Avaliação de empresas: valuation: calculando e gerenciando o valor das empresas. 3. ed. São Paulo: Pearson, 2002.

DAMODARAN, A. Damodaran on valuation: security analysis for investment and corporate finance. New York: John Wiley \& Sons, 1994.

DAMODARAN, A. Corporate finance: theory and practice. New York: John Wiley \& Sons, 1997.

DAVES, P. R.; EHRHARDT, M. C.; KUNKEL, R. A. Estimating systematic risk: the choice of return interval and estimation period. Journal of Financial and Strategic Decisions, v. 13, n. 1, p. 7-13, 2000.

DEMIGUEL, V.; GARLAPPI, L.; UPPAL, R. Optimal versus naive diversification: How inefficient is the 1/n portfolio strategy? The Review of Financial Studies, v. 22, n. 5, p. 1915-1953, 2009.

DIMSON, E. Risk measurement when shares are subject to infrequent trading. Journal of Financial Economics, v. 7, n. 2, p. 197-226, 1979.

DIMSON, E.; MARSH, P. The stability of UK risk measures and the problem of thin-trading. Journal of Finance, v. 38, n. 3, p. 753-783, 1983.

FAMA, E. F.; FRENCH, K. R. The cross-section of expected stock returns. Journal of Finance, v. 47, n. 2, p. 427-465, 1992.

FAMA, E. F.; FRENCH, K. R. Common risk factors in the returns on stocks and bonds. Journal of Financial Economics, v. 33, n. 1, p. 3-56, 1993.

FAMA, E. F.; FRENCH, K. R. Multifactor explanation of asset pricing anomalies. Journal of Finance, v. 51, n. 1, p. 55-84, 1996.

FAMA, E. F.; FRENCH, K. R. A five-factor asset pricing model. Journal of Financial Economics, v. 116, n. 1, p. 1-22, 2015.

FÁVERO, L. P. Análise de dados: modelos de regressão com $\operatorname{Excel}^{\circledR}$, Stata $^{\circledR}$ e SPSS $^{\circledR}$. Rio de Janeiro: Elsevier, 
2015.

HAGLER, C. E. M.; BRITO, R. D. O. Sobre a eficiência dos índices de ações Brasileiros. Revista de Administração, v. 42, n. 1, p. 74-85, 2007.

HANDA, P.; KOTHARI, S. P.; WASLEY, C. The relation between the return interval and betas implication for size effect. Journal of Financial Economics, v. 23, n. 1, p. 70-100, 1989.

HOECHLE, D. Robust standard errors for panel regressions with cross-sectional dependence. Stata Journal, v. 7, n. 3, p. 281-312, 2007.

JACOBS, B. I.; LEVY, K. N. Smart beta versus smart alpha. Journal of Portfolio Management, v. 40, n. 4, p. $1-4,2014$.

JEGADEESH, N.; TITMAN, S. Profitability of momentum strategies: an evaluation of alternative explanations. Journal of Finance, v. 56, n. 2, p. 699-720, 2001.

KOLLER, T.; GOEDHART, M. H.; WESSELS, D. Valuation: measuring and managing the value of companies. 4. ed. New Jersey: John Wiley \& Sons, 2005.

LAKONISHOK, J.; SHLEIFER, A.; VISHNY, R. W. Contrarian investment, extrapolation, and risk. Journal of Finance, v. 49, n. 5, p. 1541-1578, 1994.

LEVY, M.; ROLL, R. The market portfolio may be mean/variance efficient after all. The Review of Financial Studies, v. 23, n. 6, p. 2464-2491, 2010.

LINTNER, J. The Valuation of Risk Assets and the Selection of Risky Investments in Stock Portfolios and Capital Budgets. Review of Economics and Statistics, v. 47, n. 1, p. 13-37, 1965.

LIU, W. A. A liquidity-augmented capital asset pricing model. Journal of Financial Economics, v. 82, n. 3, p. 631-671, 2006.

MALKIEL, B. G. Is smart beta really smart? Journal of Portfolio Management, v. 40, n. 5, p. 127-134, 2014.

MARKOVITZ, H. M. Portfolio Selection. Journal of Finance, v. 7, n. 1, p. 77-91, 1952.

MARSH, P. Equity rights issues and the efficiency of the UK stock market. Journal of Finance, v. 34, n. 4, p. 839-862, 1979

MCLEAN, R. D.; PONTIFF, J. Does academic research destroy stock return predictability? Journal of Finance, v. 71, n. 1, p. 5-32, 2016.

NAKAMURA, W. T. Estudos Empíricos sobe a eficiência da Carteira Teórica do Índice BOVESPA. Revista de Administração do Mackenzie, v. 1, n. 1, p. 67-81, 2000.

NODA, R. F.; MARTELANC, R.; SECURATO, J. R. Eficiência da carteira de mercado no plano média-variância. Revista Brasileira de Finanças, v. 12, n. 1, p. 67-88, 2014.

PADMANABAN, N.; MUKAI, M.; TANG, L.; LE SOURD, V. Assessing the Quality of Asian Stock Market Indices, EDHEC-Risk Institute Publication, 2013.

RABELO, S. S. T. Performance das melhores práticas de governança corporativa: Um estudo de carteiras (Dissertação de Mestrado em Administração). Universidade Federal de Uberlândia, Uberlândia, Brasil, 2007.

ROLL, R. A possible explanation of the small firm effect. Journal of Finance, v. 36, n. 4, p. 879-888, 1981.

SANTOS, A. A. P.; TESSARI, C. Técnicas quantitativas de otimização de carteiras aplicadas ao mercado de ações brasileiro. Revista de Finanças Aplicadas, v. 10, n. 3, p. 369-394, 2012.

SCHAFFER, M. E.; STILLMAN, S. XTOVERID: Stata module to calculate tests of overidentifying restrictions after xtreg, xtivreg, xtivreg2, xthtaylor, 2010. <Disponível em http://ideas.repec.org/c/boc/bocode/s456779. html>

SCHOLES, M.; WILLIAMS, J. Estimating betas from nonsynchronous data. Journal of Financial Economics, v. 5 , n. 3, p. 309-327, 1977.

SCHWERT, G. W. Anomalies and market efficiency. Handbook of the Economics of Finance, 1, p. 939-974, 2003.

SERRA, R. G.; MARTELANC, R. Estimação de betas de ações com baixa liquidez. Brazilian Business Review, V. 10, n. 1, p. 49-80, 2013.

SERRA, R. G.; NAKAMURA, W. T. Is new Ibovespa the best investment option? Revista Brasileira de Gestão de Negócios, v. 18, n. 59, p. 87-107, 2016.

SERRA, R. G.; WICKERT, M. Valuation: guia fundamental. São Paulo: Atlas, 2014.

SHARPE, W. F. Capital Asset Prices: A Theory of Market Equilibrium Under Conditions of Risk. Journal of Finance, v. 19, n. 3, p. 425-442, 1964.

SHENG, H. H.; SAITO, R. Análise de métodos de replicação: O caso Ibovespa. Revista de Administração de Empresas, v. 42, n. 2, p. 66-76, 2002.

STATTMAN, D. Book values and stock returns. The Chicago MBA: A Journal of Selected Papers, v. 4, p. 2545, 1980

TAKAMATSU, R. T.; LAMOUNIER, W. M. Anúncios de prejuízos e reações dos retornos na Bovespa. In Seminários em Administração, 2006. Anais... São Paulo: SemeAd, 2006. 
THOMÉ, C. N.; LEAL, R. P. C.; ALMEIDA, V. S. Um índice de mínima variância de ações brasileiras. Economia Aplicada, v. 15, n. 4, p. 535-557, 2011. 